This is the author's version of an article published in Journal of Renewable and Sustainable Energy. Changes were made to this version by the publisher prior to publication. DOI: http://dx.doi.org/10.1063/1.4906918

\title{
Bidirectional direct current-direct current converter for fuel cell and renewable energy hybrid systems
}

\author{
Mohammad Kabalo ${ }^{1}$, Ahmed Al-Durra ${ }^{2}$, S. M. Muyeen ${ }^{2}$, and Marcelo Godoy Simôes ${ }^{3}$ \\ 1Elecsys-France 453, Rue du Breuil -Technoland 25460 Etupes - France \\ 2Department of Electrical Engineering, Petroleum Institute, Abu Dhabi, United Arab Emirates \\ 3Electrical Engineering and Computer Science Department, Colorado School of Mines, 1610 Illinois St. \\ Golden, Colorado 804010-1887, USA
}

\begin{abstract}
A bidirectional dc-dc power converter is an essential part of a HFCEV (Hybrid Fuel Cell Electric Vehicle) and renewable energy hybrid system. This paper presents a new bidirectional dc-dc power converter for HFCEV and renewable energy hybrid systems. The proposed converter is called four phases floating interleaved boost converter. Two control strategies of the dc-bus voltage are discussed. In the first one, a dual loop with linear current and voltage controllers is developed, based on the frequency response of the system. In the second one, the linear current controller is replaced by a non-linear sliding mode current controller, to improve the system performance from the robustness point of view. The non-linear sliding mode controller is designed based on the average model of the proposed topology. The proposed control strategies have been validated by simulation and experimental results.
\end{abstract}

\section{INTRODUCTION}

Electric vehicle technology has been adopting fuel cell (FC) for hybrid applications in the last few years. Therefore, the bidirectional dc-dc power converter is fundamental for the hybridization of fuel cell vehicle, with on-board energy management for achieving high performance systems. ${ }^{1-4}$ The bidirectional dc-dc converter is also an important part of many renewable energy hybrid applications, such as photovoltaic (PV) generation systems and wind power systems. $^{5-11}$ Figure 1 shows the Hydrogen FC Electric Vehicle (HFCEV) and the renewable energy hybrid system architecture.

In the HFCEV configuration, the battery is used to provide the peak power demand during the acceleration phase, and to recover the energy during the breaking phase. The fuel cell current is controlled by the unidirectional dc-dc converter aiming to force the fuel cell to work in its optimal zone (the zone where the efficiency is maximum). In the HFCEV, the vehicle average power is provided by the fuel cell. In the renewable energy hybrid system, the role of the battery is to stock the energy when the production of the PV module is higher than the load demand, and to provide it in the opposite case.

In the two applications, the HFCEV and the renewable energy hybrid system, the dc-bus voltage is controlled by the bidirectional dc-dc converter. 


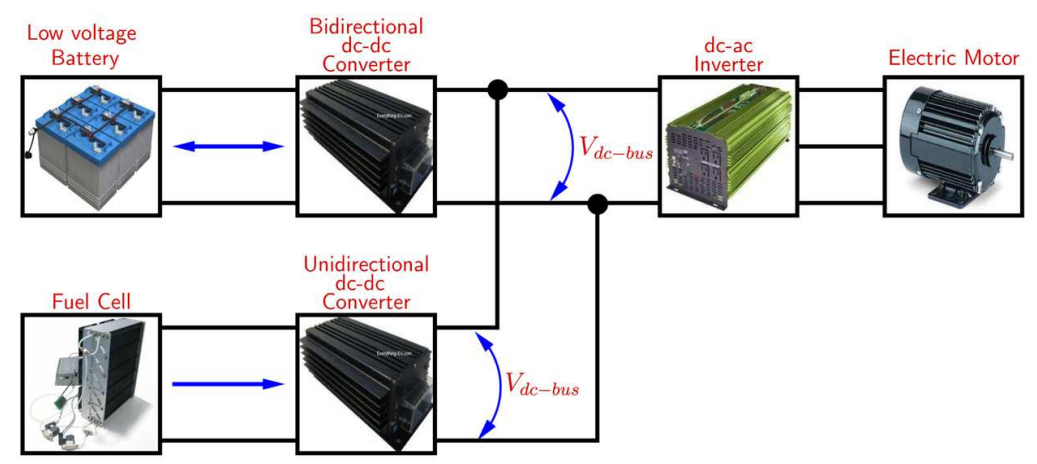

(a)

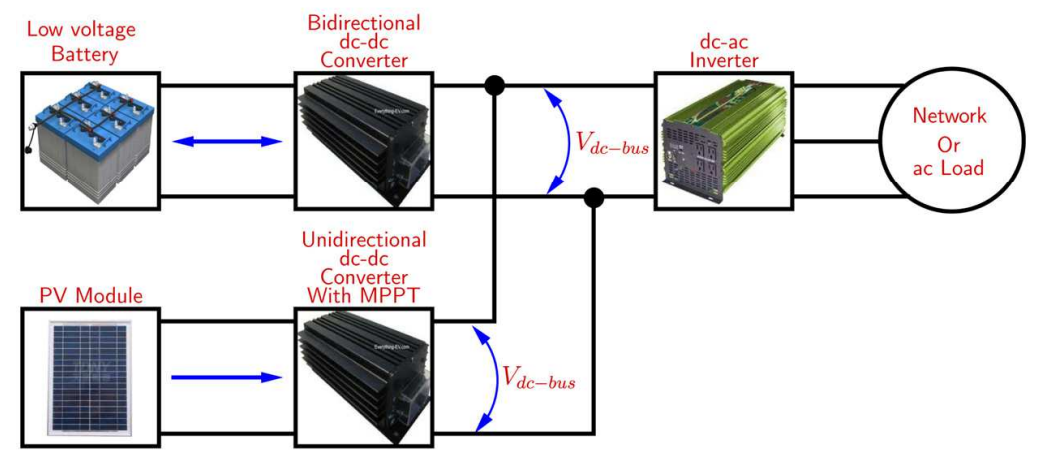

(b)

FIG. 1. HFCEV and renewable energy hybrid system architecture. (a) HFCEV architecture and (b) Renewable energy hybrid system architecture.

The dc-bus voltage of HFCEV and renewable energy hybrid system is a high-voltage of a few hundred volts. This means that the voltage difference between the battery and the dc-bus is large; thus, the bidirectional dc-dc converter should have a high step-up conversion ratio. A cascade DC-DC converter composed of two phase interleaved boost converter and three level series boost converter is proposed in Ref. 12. This solution suffers from low efficiency and poor reliability. In Refs. 13 and 14, a parallel resonant converter with a capacitor as output filter is proposed. However, in this topology the determination of the leakage inductance and capacitance is very complex as well as the topology modeling.

Figure 2 presents the configuration of the proposed bidirectional 4-phase floating interleaved boost converter (FIBC) converter. The proposed bidirectional 4-phase FIBC is composed from two non-floating and two floating modules, with parallel connection at the input and series connection at the output, aiming to achieve high voltage ratio, low input current ripple, and high efficiency.

The floating output with interleaved input allows reduction of current rating and voltage stresses for the semiconductors, making this topology more cost effective and more efficient than the conventional boost and interleaved boost converter. ${ }^{15-17}$ The advantages of proposed topology related to the high conversion ratio, high efficiency, capacitor and inductor volume reduction, RMS capacitor current reduction, input current ripple reduction, and power electronic devices rating reduction have been reported in Refs. 18 and 19. The operation principles and sizing of the proposed converter in unidirectional configuration have been reported in Ref. 20. In Ref. 20, a different HFCEV architecture is studied, where a high voltage battery of $400 \mathrm{~V}$ is directly connected to the dc-bus imposing its voltage. Therefore, a single loop linear current controller is used to control the current of the fuel cell in order to force it to operate in its optimal zone. 


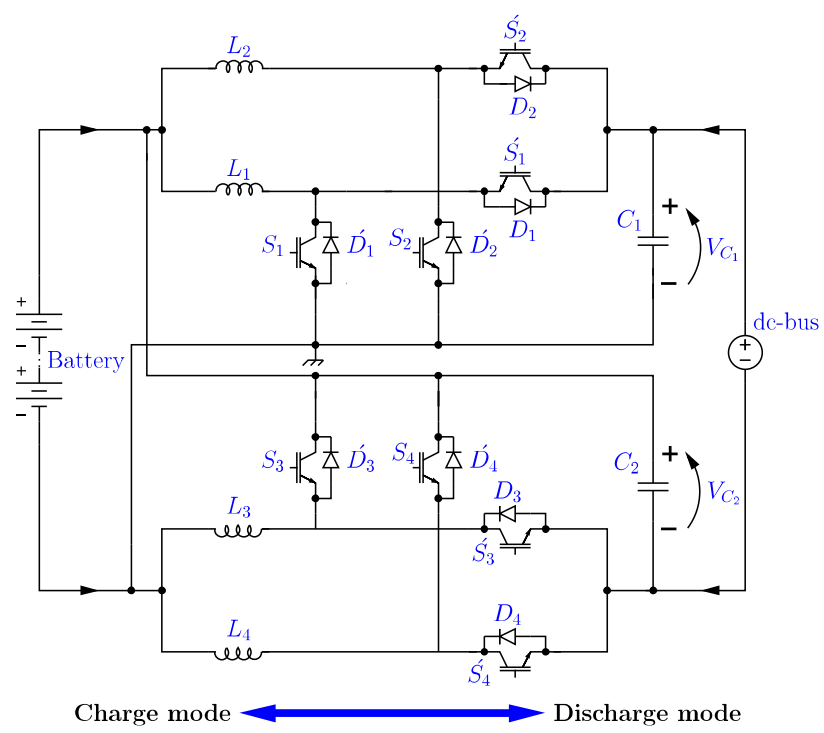

FIG. 2. Proposed bidirectional 4-phase FIBC converter.

\section{DC-BUS VOLTAGE CONTROL STRATEGIES}

In this paper, two control strategies of the dc-bus voltage of the HFCEV and the renewable energy hybrid system will be presented. A dual loop control with linear current and voltage controllers will be first developed. A hybrid dual loop control with a linear voltage controller and a non-linear sliding mode current controller will also be developed. The two control strategies will be analyzed in order to achieve the desired performance of the system.

\section{A. First control strategy}

This control strategy uses two loops, an inner inductors current loop where a linear inductors current controller $G_{c i}(s)$ is used, and an outer dc-bus voltage loop where a linear dc-bus voltage controller $G_{c v}(s)$ is used as shown in Figure 3.

The dc-bus voltage can be controlled using a single loop voltage controller (classical method). However, this method leads to inequivalent current sharing between the phases since the current in each phase is not controlled. This situation leads to damage the passive and the active components of the converter, since they are designed to support the same phase current. Therefore, inductors current loop must be used to avoid such situation.

The frequency response technique is used to design the dc-bus voltage controller and the inductors current controller (determination of the fundamental parameters, which are the phase margin (PM), the crossover frequency $f_{c}$ and the gain at low frequency). The gains of the inner inductors current loop $T_{i}(s)$ and the outer dc-bus voltage loop $T_{v}(s)$ are given in the following equations:

$$
T_{i}(s)=G_{c i}(s) G_{p}(s) G_{d i_{L}}(s) H_{f i}(s),
$$

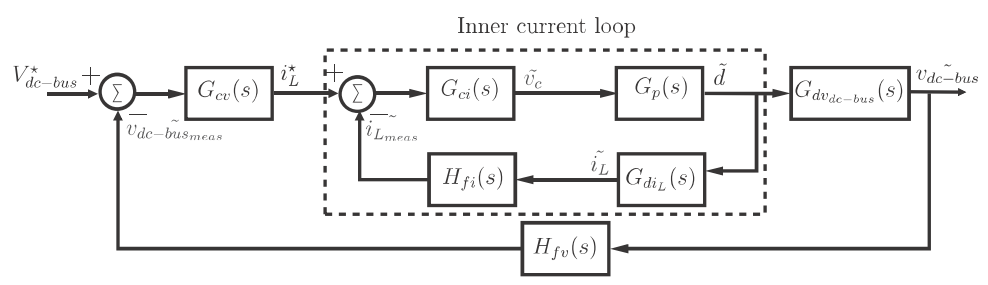

FIG. 3. Dual loop control strategy structure. 


$$
T_{v}(s)=\frac{G_{c v}(s) G_{c i}(s) G_{p}(s) G_{d v_{d c-b u s}}(s) H_{f v}(s)}{1+G_{c i}(s) G_{p}(s) G_{d i_{L}}(s) H_{f i}(s)}
$$

where $H_{f i}(s), H_{f v}(s)$ are the transfer functions of the inductors current and the dc-bus voltage measurement filter $\left(K_{f i}=K_{f v}=1\right.$ and $T_{f i}=T_{f v}=22 \mu \mathrm{s}$ (getting a cutoff frequency of $7 \mathrm{kHz}$ )). $G_{p}(s)$ is the pulse-width modulator transfer function. $G_{d v_{d c-b u s}}(s)$ and $G_{d i_{L}}(s)$ are the transfer functions of the duty-cycle to the dc-bus voltage and the duty-cycle to the inductors current. The two transfer functions are given by the following equations: ${ }^{20}$

$$
\begin{gathered}
G_{d v_{d c-b u s}}(s)=\frac{v_{d c-\tilde{b u s}}(s)}{\tilde{d}(s)}=K_{v} \frac{\left(1-\frac{s}{w_{z r}}\right)\left(1+\frac{s}{w_{z l}}\right)}{\left(1+\frac{s}{w_{o} Q}+\frac{s^{2}}{w_{o}^{2}}\right)}, \\
G_{d i_{L}}(s)=\frac{\tilde{i_{L}}(s)}{\tilde{d}(s)}=K_{i} \frac{\left(1+\frac{s}{w_{z i}}\right)}{\left(1+\frac{s}{w_{o} Q}+\frac{s^{2}}{w_{o}^{2}}\right)},
\end{gathered}
$$

where

$$
\begin{gathered}
K_{v}=\frac{V_{b}\left(2 R(1-\delta)^{2}-R_{L}(1+\delta)\right)}{(1-\delta)^{2}\left(R(1-\delta)^{2}+R_{L}\right)}, \\
w_{z r}=\frac{2 R(1-\delta)^{2}-R_{L}(1+\delta)}{L(1+\delta)}, \\
w_{z l}=\frac{1}{R_{C} C}, \\
K_{i}=\frac{V_{b}(\delta+3)}{2(1-\delta)\left(R(1-\delta)^{2}+R_{L}\right)}, \\
w_{z i}=\frac{1}{\frac{R C}{\delta+3}+R_{C} C}, \\
w_{o}=\frac{1}{\sqrt{L C}} \sqrt{\frac{2 R(1-\delta)^{2}+2 R_{L}}{R+2 R_{C}}}, \\
R C\left(R_{L}+2 R_{C}(1-\delta)^{2}\right)+2\left(L+R_{C} R_{L} C\right)
\end{gathered} .
$$

The transfer functions (3) and (4) construct a system of second-order with corner frequency $w_{o}$ and double-pole, ESR (Equivalent Series Resistance) zero $w_{z l}$ and RHP (Right Half Plane) zero $w_{z r}$.

The specifications of the system used in this study are given in Table I.

Figure 4 presents the frequency response of the compensated and uncompensated voltage and current loop. The very low gain at low frequency of the uncompensated current loop leads to important steady state error. Therefore, a lag controller has to be used to cancel the steady state error and to reduce the system time response. The parameters of the lag controller of the current loop are determined to get a PM of $68^{\circ}$ (a PM in a range of $45^{\circ}-90^{\circ}$ is desirable ${ }^{21,22}$ ) and a crossover frequency of $800 \mathrm{~Hz}$. 
TABLE I. Specifications of the system.

\begin{tabular}{lc}
\hline \hline Parameters & Value \\
\hline Battery voltage & $V_{b}=72 \mathrm{~V}$ \\
Rated power & $P_{R}=5 \mathrm{~kW}$ \\
Voltage of the DC-bus & $V_{d c-b u s}=400 \mathrm{~V}$ \\
Capacitor value & $C=2 \mathrm{mF}$ \\
ESR of the capacitor & $R_{C}=20 \mathrm{~m} \Omega$ \\
Inductor value & $L=100 \mu \mathrm{H}$ \\
ESR of the inductor & $R_{L}=40 \mathrm{~m} \Omega$ \\
Switching frequency & $f=20 \mathrm{kHz}$ \\
Load resistor & $R=32 \Omega$ \\
\hline \hline
\end{tabular}

By using a lag controller, the gain at low frequency of the uncompensated voltage loop can also be improved, which permits to get a response with neglected steady state error. The voltage loop should have a bandwidth lower than the current loop to prevent the interaction between the two controllers. The parameters of the lag controller of the voltage loop are determined to get a PM of $78^{\circ}$ and a crossover frequency of $300 \mathrm{~Hz}$. Equations (5) and (6) give the transfer functions of the voltage and current controllers.

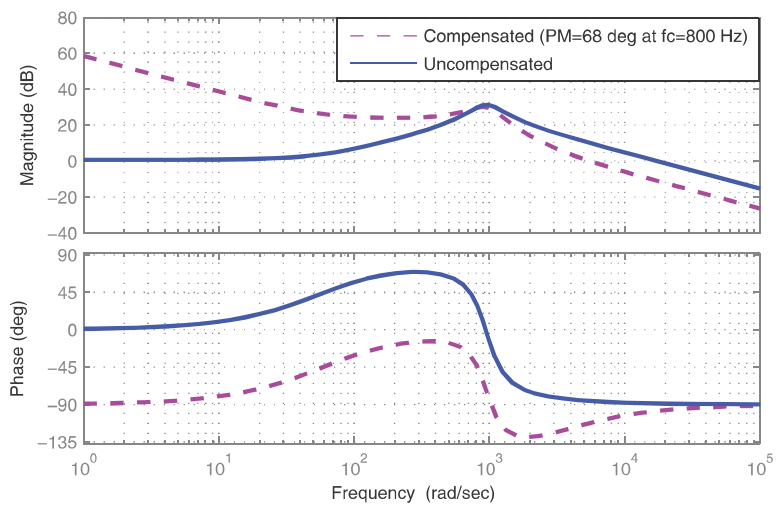

(a)

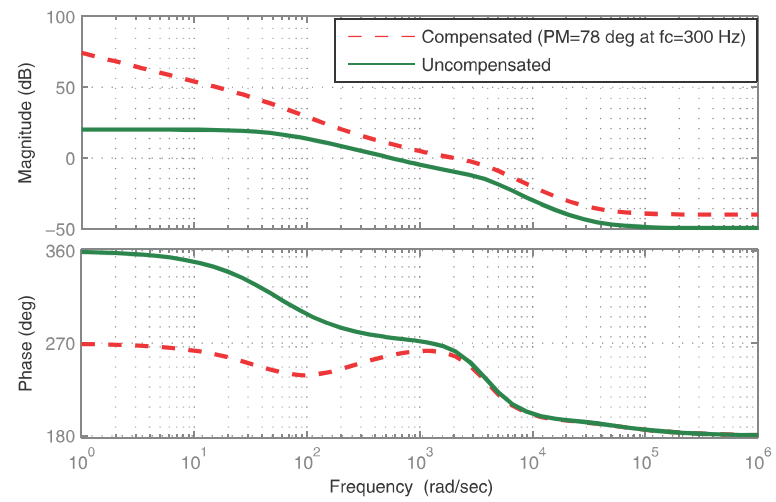

(b)

FIG. 4. Bode diagram of uncompensated and compensated voltage and current loop. (a) Bode diagram of uncompensated and compensated current loop. (b) Bode diagram of uncompensated and compensated voltage loop. 


$$
\begin{gathered}
G_{c i}(s)=0.002\left(1+\frac{2750}{s}\right), \\
G_{c v}(s)=3\left(1+\frac{167}{s}\right) .
\end{gathered}
$$

In HFCEV and renewable energy hybrid system, the bidirectional 4-phase FIBC will work with different scenarios of power demand. These scenarios vary from light power demand (when the power demand is equal to the power provided by the fuel cell or by the PV module) to important power demand (when the power demand is higher than the power provided by the fuel cell or by the PV module). Therefore, it is necessary to evaluate the performance of the first control strategy according to these scenarios of load variations. The frequency response, at different power demand, of the voltage and current loop is given in Figure 5.

From Figure 5, one can see that the gain at low frequency of the voltage loop becomes more important at light power demand. This means that the system performance will not be affected from the steady state error point of view. On the other hand, the current loop low frequency gain becomes smaller when decreasing the load. Therefore, the system becomes less efficient from the steady state error and time response point of view. So, another controller has to be used where the load variations have no effects on the global system performances. In

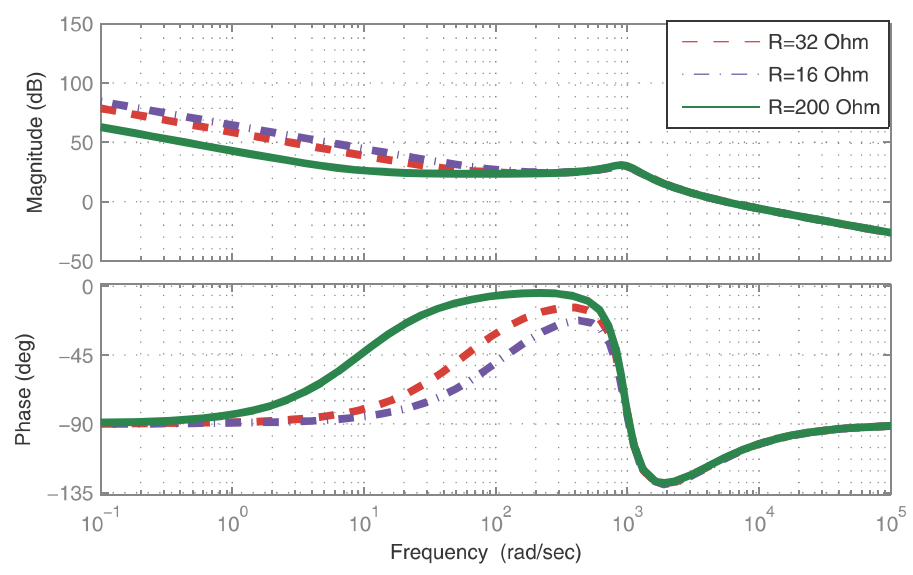

(a)

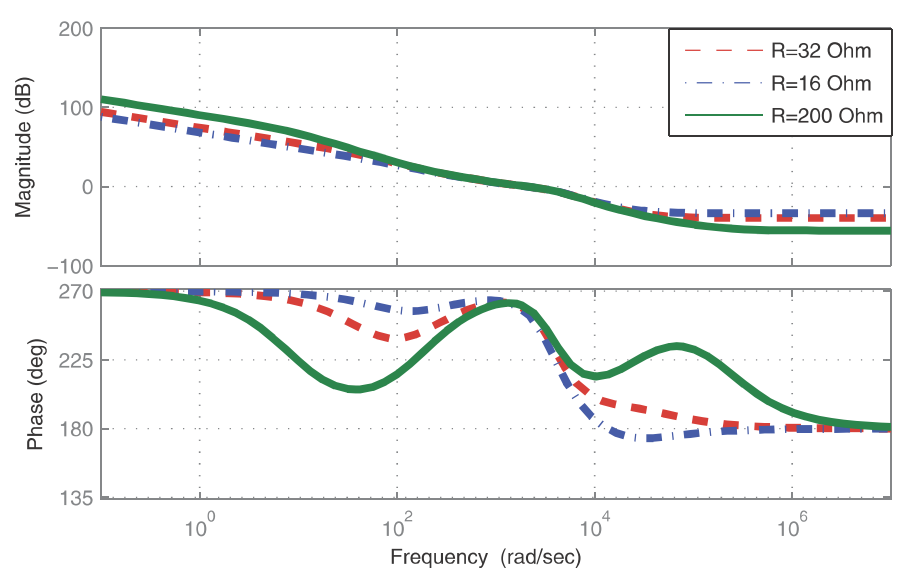

(b)

FIG. 5. Bode diagram of voltage and current loop. (a) Bode diagram of current loop. (b) Bode diagram of voltage loop. 
the second control strategy, the authors proposed replacing the linear current controller by a non-linear sliding mode controller.

\section{B. Second control strategy}

In this control strategy, a linear voltage controller is used to generate the inductors current reference, whereas the control inputs are generated using a non-linear sliding mode controllers as shown in Figure 6.

The average model, with the different states and control variables, of the bidirectional 4phase FIBC in boost operation (discharge mode) is defined in the following equation:

$$
\begin{aligned}
& {\left[\begin{array}{c}
\dot{I_{L_{1}}} \\
\dot{I_{L_{2}}} \\
\dot{I_{L_{3}}} \\
\dot{I_{L_{4}}} \\
\dot{V_{C_{1}}} \\
\dot{V_{C_{2}}}
\end{array}\right]=\left[\begin{array}{ccccccc}
\left(\frac{\delta_{1}+1}{2 L_{1}}\right) & \left(\frac{\delta_{1}-1}{2 L_{1}}\right) & \left(\frac{-R_{L_{1}}}{L_{1}}\right) & 0 & 0 & 0 & 0 \\
\left(\frac{\delta_{2}+1}{2 L_{2}}\right) & \left(\frac{\delta_{2}-1}{2 L_{2}}\right) & 0 & \left(\frac{-R_{L_{2}}}{L_{2}}\right) & 0 & 0 & 0 \\
\left(\frac{\delta_{3}+1}{2 L_{3}}\right) & \left(\frac{\delta_{3}-1}{2 L_{3}}\right) & 0 & 0 & \left(\frac{-R_{L_{3}}}{L_{3}}\right) & 0 & 0 \\
\left(\frac{\delta_{4}+1}{2 L_{4}}\right) & \left(\frac{\delta_{4}-1}{2 L_{4}}\right) & 0 & 0 & 0 & \left(\frac{-R_{L_{4}}}{L_{4}}\right) & 0 \\
0 & 0 & \left(\frac{1-\delta_{1}}{C_{1}}\right) & \left(\frac{1-\delta_{2}}{C_{1}}\right) & 0 & 0 & \left(\frac{-1}{C_{1}}\right) \\
0 & 0 & 0 & 0 & \left(\frac{1-\delta_{3}}{C_{2}}\right) & \left(\frac{1-\delta_{4}}{C_{2}}\right) & \left(\frac{-1}{C_{2}}\right)
\end{array}\right]} \\
& \times\left[\begin{array}{c}
V_{b} \\
V_{d c-b u s} \\
I_{L_{1}} \\
I_{L_{2}} \\
I_{L_{3}} \\
I_{L_{4}} \\
I_{d c-b u s}
\end{array}\right] .
\end{aligned}
$$

Sliding control law surfaces can be defined as given in the following equation:

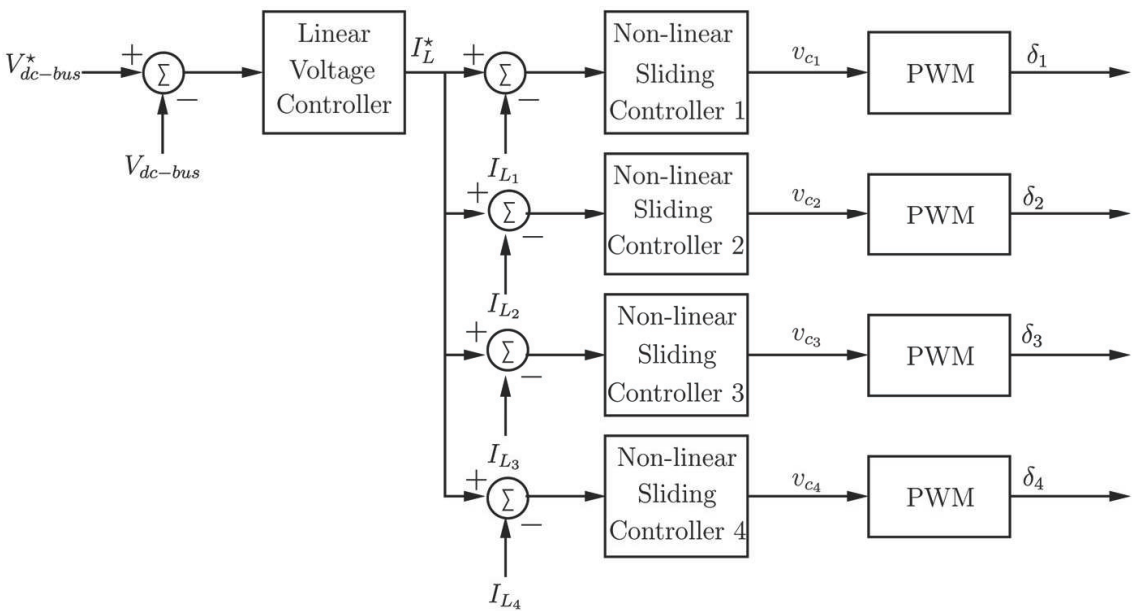

FIG. 6. Structure of the second control strategy. 


$$
\left[\begin{array}{c}
S_{I_{L 1}} \\
S_{I_{L 2}} \\
S_{I_{L 3}} \\
S_{I_{L 4}}
\end{array}\right]=\left[\begin{array}{c}
I_{L_{1}}-I_{L_{1}}^{\star}+\kappa_{I_{L_{1}}} \int_{0}^{t}\left(I_{L_{1}}-I_{L_{1}}^{\star}\right) d \tau \\
I_{L_{2}}-I_{L_{2}}^{\star}+\kappa_{I_{L_{2}}} \int_{0}^{t}\left(I_{L_{2}}-I_{L_{2}}^{\star}\right) d \tau \\
I_{L_{3}}-I_{L_{3}}^{\star}+\kappa_{I_{L_{3}}} \int_{0}^{t}\left(I_{L_{3}}-I_{L_{3}}^{\star}\right) d \tau \\
I_{L_{4}}-I_{L_{4}}^{\star}+\kappa_{I_{4}} \int_{0}^{t}\left(I_{L_{4}}-I_{L_{4}}^{\star}\right) d \tau
\end{array}\right],
$$

where $I_{L_{1}}^{\star}$ to $I_{L_{4}}^{\star}$ are the desired inductors current. The convergence to zero of the static error is determined according to the coefficients $\kappa_{I_{1}}$ to $\kappa_{I_{L_{4}}}$. The sliding surfaces dynamic convergence to zero is given in the following equation:

$$
\left[\begin{array}{l}
\dot{S_{I_{L 1}}} \\
\dot{S_{I_{L 2}}} \\
\dot{S_{I_{L 3}}} \\
\dot{S_{I_{L 4}}}
\end{array}\right]=\left[\begin{array}{cccc}
-\lambda_{I_{L_{1}}} & 0 & 0 & 0 \\
0 & -\lambda_{I_{L_{2}}} & 0 & 0 \\
0 & 0 & -\lambda_{I_{L_{3}}} & 0 \\
0 & 0 & 0 & -\lambda_{I_{L_{4}}}
\end{array}\right]\left[\begin{array}{l}
S_{I_{L 1}} \\
S_{I_{L 2}} \\
S_{I_{L 3}} \\
S_{I_{L 4}}
\end{array}\right],
$$

where $\lambda_{I_{L_{1}}}$ to $\lambda_{I_{L_{4}}}$ are the convergence factors (positive real numbers).

By combining Eqs. (7)-(9), the control inputs can be obtained as given in the following equation:

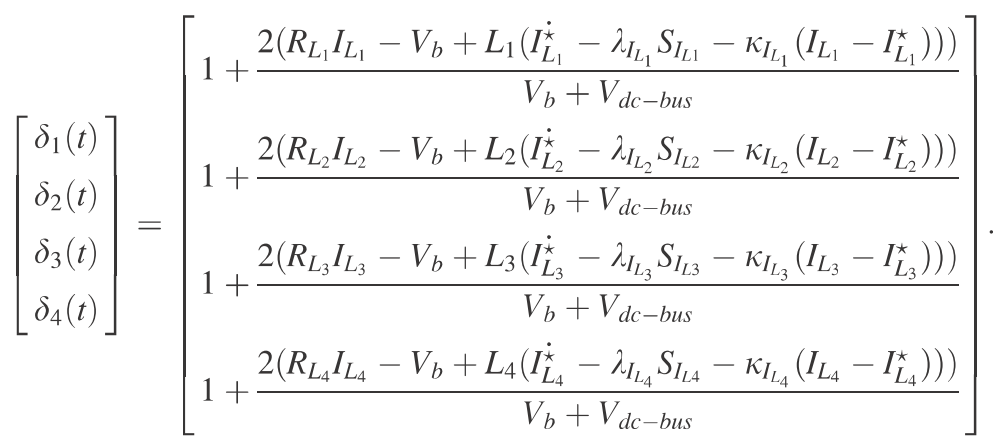

From Eq. (10), one can see that the independence of the control inputs of the load $R$, justifies the robustness of this control strategy from the load variations point of view.

By replacing the control inputs in the average model of the 4-phase FIBC, we get the following equation:

$$
\left[\begin{array}{l}
\dot{x}_{1}+\left(\kappa_{I_{L_{1}}}+\lambda_{I_{L_{1}}}\right) x_{1}+\kappa_{I_{L_{1}}} \lambda_{I_{L_{1}}} \int_{0}^{t} x_{1} d \tau \\
\dot{x}_{2}+\left(\kappa_{I_{L_{2}}}+\lambda_{I_{L_{2}}}\right) x_{2}+\kappa_{I_{L_{2}}} \lambda_{I_{L_{2}}} \int_{0}^{t} x_{2} d \tau \\
\dot{x}_{3}+\left(\kappa_{I_{L_{3}}}+\lambda_{I_{L_{3}}}\right) x_{3}+\kappa_{I_{L_{3}}} \lambda_{L_{L_{3}}} \int_{0}^{t} x_{3} d \tau \\
\dot{x}_{4}+\left(\kappa_{I_{L_{4}}}+\lambda_{I_{L_{4}}}\right) x_{4}+\kappa_{I_{L_{4}}} \lambda_{I_{L_{4}}} \int_{0}^{t} x_{4} d \tau
\end{array}\right]=\left[\begin{array}{l}
0 \\
0 \\
0 \\
0
\end{array}\right],
$$

where

$$
\left[\begin{array}{l}
x_{1} \\
x_{2} \\
x_{3} \\
x_{4}
\end{array}\right]=\left[\begin{array}{c}
I_{L_{1}}-I_{L_{1}}^{\star} \\
I_{L_{2}}-I_{L_{2}}^{\star} \\
I_{L_{3}}-I_{L_{3}}^{\star} \\
I_{L_{4}}-I_{L_{4}}^{\star}
\end{array}\right]
$$

The system can be represented by the following equation: 


$$
\left[\begin{array}{l}
\ddot{x}_{1}+\left(\kappa_{I_{L_{1}}}+\lambda_{I_{L_{1}}}\right) \dot{x}_{1}+\kappa_{I_{L_{1}}} \lambda_{I_{L_{1}}} x_{1} \\
\ddot{x}_{2}+\left(\kappa_{I_{L_{2}}}+\lambda_{I_{L_{2}}}\right) \dot{x}_{2}+\kappa_{I_{L_{2}}} \lambda_{I_{L_{2}}} x_{2} \\
\ddot{x}_{3}+\left(\kappa_{I_{L_{3}}}+\lambda_{I_{L_{3}}}\right) \dot{x}_{3}+\kappa_{I_{L_{3}}} \lambda_{I_{L_{3}}} x_{3} \\
\ddot{x}_{4}+\left(\kappa_{I_{L_{4}}}+\lambda_{I_{L_{4}}}\right) \dot{x}_{4}+\kappa_{I_{L_{4}}} \lambda_{I_{L_{4}}} x_{4}
\end{array}\right]=\left[\begin{array}{l}
0 \\
0 \\
0 \\
0
\end{array}\right]
$$

From Eq. (13), one can see that the final response is independent of the topology parameters. This leads to the fact that the final response will not be affected by the variations of these parameters. The stability of the system is ensured since all the coefficients in Eq. (13) are positive.

The average model of the proposed converter in buck operating (charge mode) can be defined as given in the following equation:

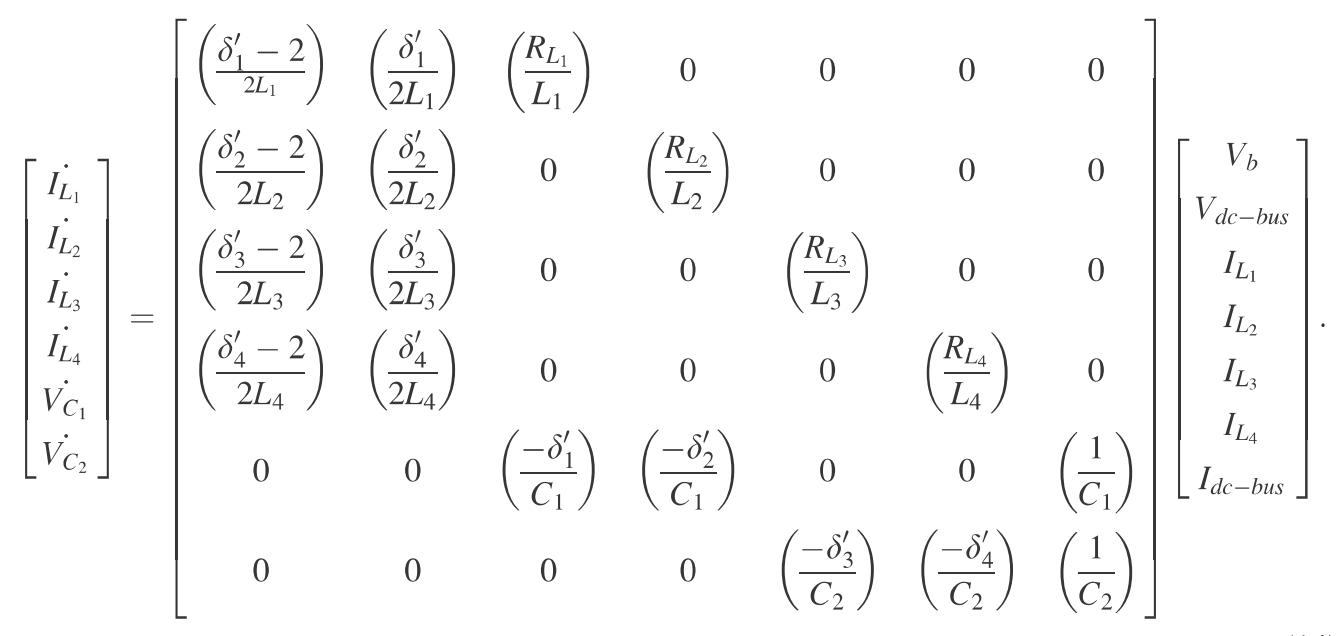

$\delta^{\prime}$ is the duty cycle of conduction of the switches $\left(S_{1}^{\prime}, S_{2}^{\prime}, S_{3}^{\prime}, S_{4}^{\prime}\right)$ in buck operation. $\delta^{\prime}$ is also the duty cycle of conduction of the diodes $\left(D_{1}, D_{2}, D_{3}, D_{4}\right)$ in boost operation (discharge mode). If $\delta$ is the duty cycle of conduction of the switches $\left(S_{1}, S_{2}, S_{3}, S_{4}\right)$ in boost operation, one can write

$$
\delta^{\prime}=1-\delta
$$

Using this equation, one can see that the difference between the average model of the proposed converter in buck operation and that in boost operation is only the sign of the current, which is reversed. However, as this current in buck operation was chosen in the opposite direction from that in boost operation, the two models are identical. Therefore, the bidirectional 4-phase FIBC can use the same current controller for the two modes of operation.

In the bidirectional 4-phase FIBC, the switches $\left(S_{1}^{\prime}, S_{2}^{\prime}, S_{3}^{\prime}, S_{4}^{\prime}\right)$ are commanded with the complementary signals of the switches $\left(S_{1}, S_{2}, S_{3}, S_{4}\right)$. Therefore, a dead time between $\left(S_{1}, S_{1}^{\prime}\right)$, $\left(S_{2}, S_{2}^{\prime}\right),\left(S_{3}, S_{3}^{\prime}\right)$, and $\left(S_{4}, S_{4}^{\prime}\right)$ is needed to avoid the short circuit.

\section{SIMULATION RESULTS}

The second control strategy is validated by a simulation results as shown in Figure 7.

From Figure 7, it can be observed that the voltage controller sets the dc-bus voltage at its reference, and the non-linear sliding controllers force the inductors current to follow perfectly their reference generated by the linear voltage controller, without any oscillations or overshoot. 


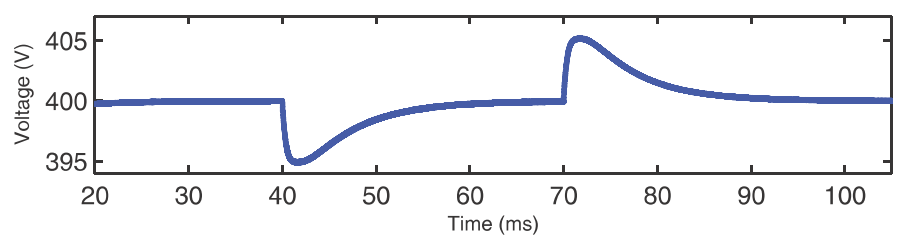

(a)

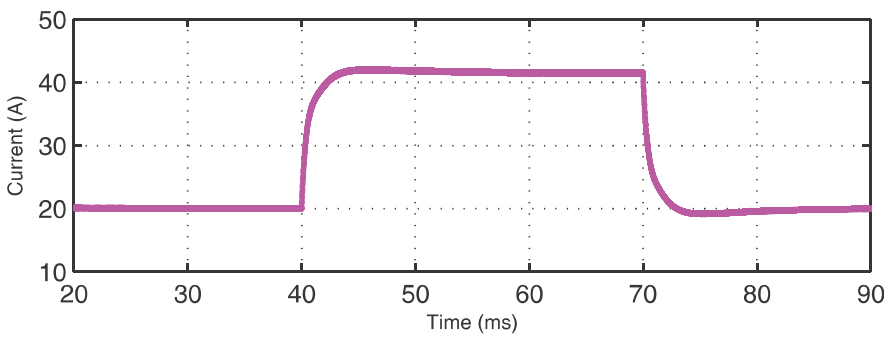

(b)

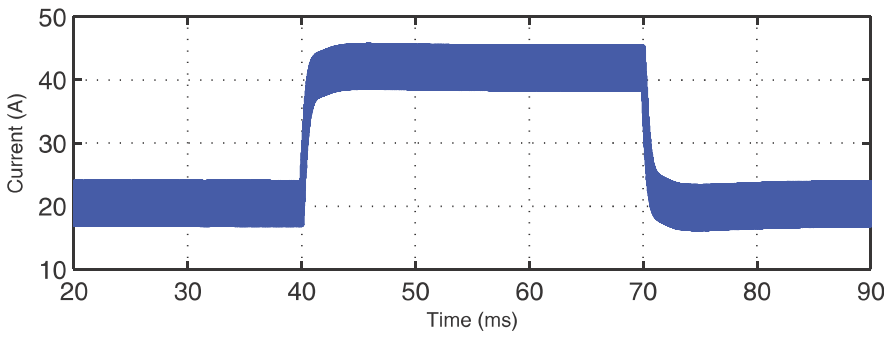

(c)

FIG. 7. Second control strategy response when important changes of load are applied (increasing power with $100 \%$ and then coming back to rated power). (a) dc-Bus voltage $\left(V_{d c-b u s}^{\star}=400 \mathrm{~V}\right)$. (b) Voltage controller response $\left(I_{L}^{\star}\right)$. (c) $I_{L_{1}}$ to $I_{L_{4}}$.

The response of the second control strategy during a change of mode of operation (from discharge to charge mode) is shown in Figure 8. It can be observed that the bidirectional 4phase FIBC can operate in boost and buck operation using the same current controllers, without any current oscillations or overshoot and with high dynamic performance.

\section{EXPERIMENTAL RESULTS}

The realized converter is shown in Figure 9.

Figures 10 and 11 present the experimental results when an important change of power demand is applied to verify the reaction of the second control strategy.

One can see that the voltage controller keeps the dc-bus voltage at its desired value, regardless of the changes of power demand. On the other hand, one can also see that the current controllers set the inductors current at their desired value. This performance justifies the robustness of the second control strategy under a wide range of load variations.

The steady state waveforms of the bidirectional 4-phase FIBC for $I_{L}^{\star}=2 \mathrm{~A}$ are illustrated in Figure 12.

From Figure 12, one can observe that the input current is always positive with neglected ripple, whereas the inductors current have negative part. This permits to use the same current controller regardless of the average value of the inductors current and the mode of operation (charge or discharge mode). 


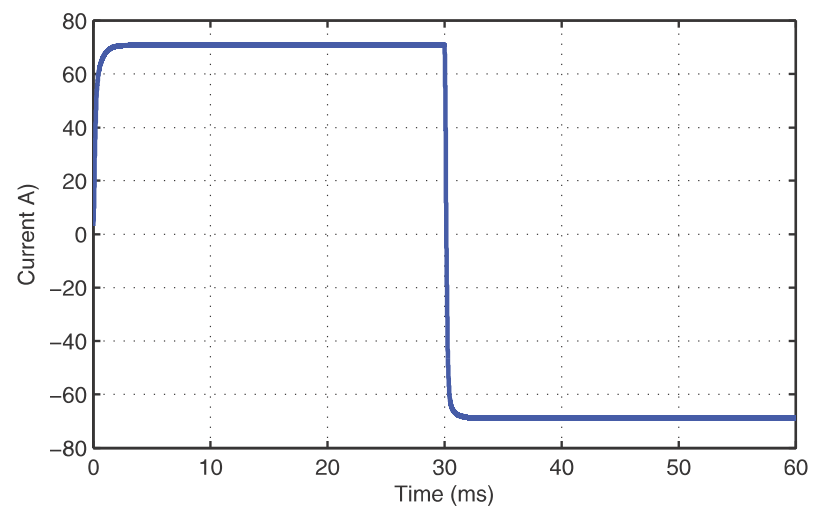

(a)

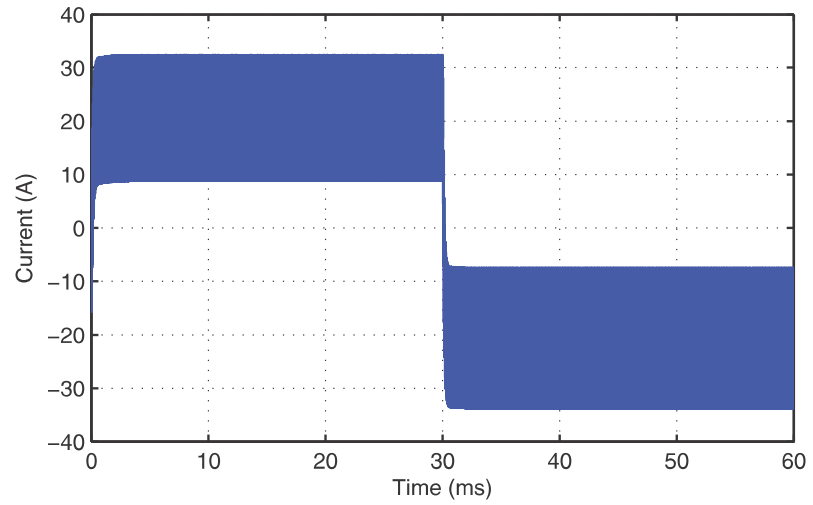

(b)

FIG. 8. Battery and inductors current during the change of mode of operation. (a) Battery current during the change of mode of operation ( $I_{L}^{\star}$ from $20.6 \mathrm{~A}$ to $-20.6 \mathrm{~A}$ ). (b) Inductors current during the change of mode of operation $\left(I_{L}^{\star}\right.$ from $20.6 \mathrm{~A}$ to $-20.6 \mathrm{~A})$

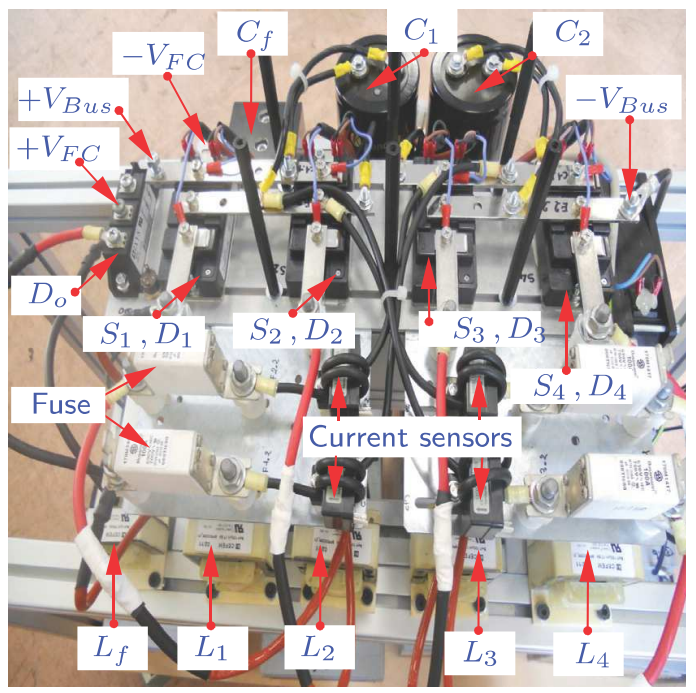

FIG. 9. Realized bidirectional 4-phase FIBC converter 


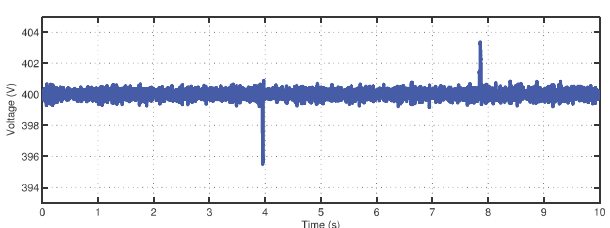

(a)

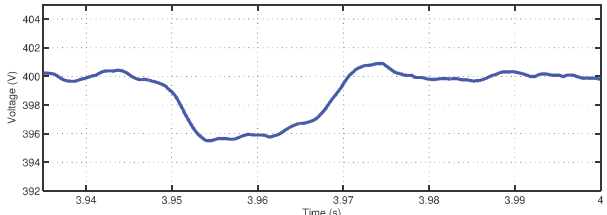

(b)

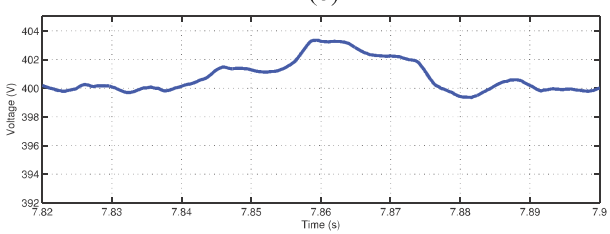

(c)

FIG. 10. Second control strategy validation (voltage controller performance). (a) Voltage controller performance $\left(V_{d c-b u s}^{\star}=400 \mathrm{~V}\right)$. (b) Voltage controller performance when changing the load. (c) Voltage controller performance when return to the nominal load.

\section{CONCLUSION}

In this paper a bidirectional 4-phase FIBC for fuel cell and renewable energy hybrid systems has been presented. Two control strategies of the dc-bus voltage of the proposed converter have been developed. Initially, a dual loop control strategy has been developed, with linear current and dc-bus voltage controllers. However, the frequency response analysis of the dual loop control strategy has been shown that the inductor current loop will suffer from poor performance when one passes from full load to light load conditions. Therefore, a non-linear sliding mode controller is used for the current loops, which results in a hybrid dual loop control strategy with linear controller for the dc-bus voltage and non-linear controller for the inductors current. The second control strategy has been shown high robustness for a wide range of load

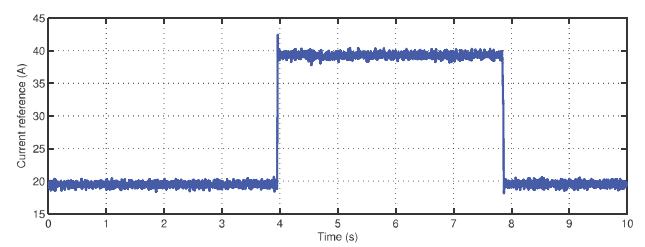

(a)

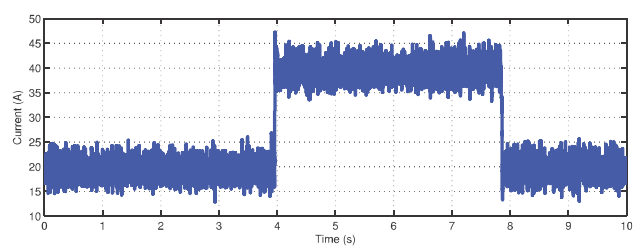

(b)

FIG. 11. Second control strategy validation (current controller performance). (a) Current reference (voltage controller response). (b) Current controller performance when changing the load. 


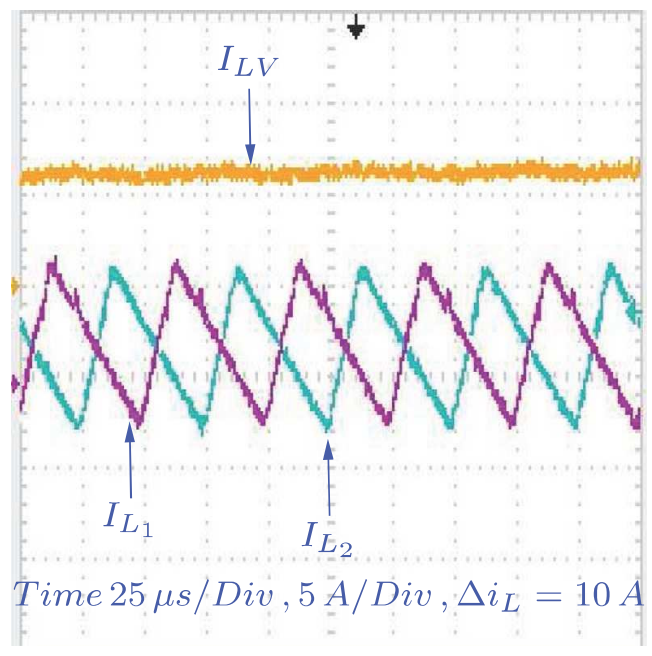

(a)

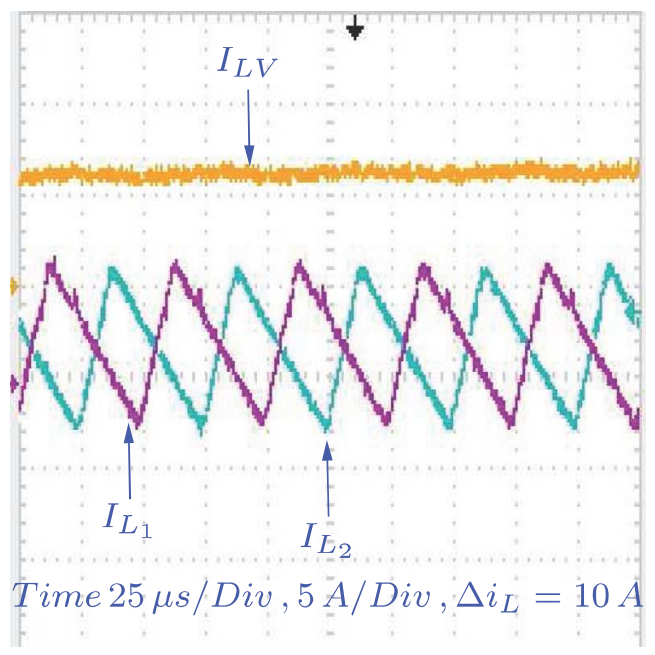

(b)

FIG. 12. Battery and inductors current for $I_{L}^{\star}=2 \mathrm{~A}$. (a) Battery and inductors current $\left(I_{L_{1}}, I_{L_{2}}\right)$ for $I_{L}^{\star}=2 \mathrm{~A}$. (b) Battery and inductors current $\left(I_{L_{3}}, I_{L_{4}}\right)$ for $I_{L}^{\star}=2 \mathrm{~A}$.

variations. Simulation and experimental results have been validated the second control strategy. The proposed bidirectional converter with the developed control strategy demonstrates a lot of potential and promises for utilization in fuel cell and renewable energy hybrid systems.

${ }^{1}$ M. Kabalo, B. Blunier, D. Bouquain, and A. Miraoui, in IEEE Vehicle Power and Propulsion Conference (VPPC) Lille, France, 1-3 September, 2010.

${ }^{2}$ K.-H. Chao and C.-H. Huang, IET Power Electron. 7, 1557 (2014).

${ }^{3}$ R.-J. Wai, L.-S. Hong, and J.-J. Liaw, IET Power Electron. 7, 1278 (2014).

${ }^{4} \mathrm{H}$. Do and J. Yang, IEEE Trans. Ind. Electron. 61, 3248 (2014).

${ }^{5}$ W. Li, C. Xu, H. Yu, Y. Gu, and X. He, IET Power Electron. 7, 67 (2014).

${ }^{6}$ P. W. L. K. Filsoof and A. A. Hagar, IET Power Electron. 7, 2190 (2014).

${ }^{7}$ Z.-H. Ding, C. Yang, Z. Zhang, C. Wang, and S.-J. Xie, IEEE Trans. Power Electron. 29, 1595 (2014).

${ }^{8}$ B. Zhao, Q. Song, and W. Liu, IEEE Trans. Ind. Electron. 61, 1389 (2014).

${ }^{9}$ A. F. M. R. Banaei and H. Ardi, IET Power Electron. 7, 1906 (2014).

${ }^{10}$ B. Zhao, Q. Song, W. Liu, and S. Yandong, IEEE Trans. Ind. Electron. 61, 5402 (2014).

${ }^{11}$ D. Han, J. Noppakunkajorn, and B. Sarlioglu, IEEE Trans. Veh. Technol. 63, 3001 (2014).

${ }^{12}$ A. Shahin, M. Hinaje, J. Martin, S. Pierfederici, S. Rael, and B. Davat, IEEE Trans. Ind. Electron. 57, 3944 (2010).

${ }^{13}$ J. Martin-Ramos, J. Diaz, A. Pernia, J. Lopera, and F. Nuno, IEEE Trans. Ind. Electron. 54, 2262 (2007).

${ }^{14} \mathrm{~J}$. Martin-Ramos, A. Pernia, J. Díaz, F. Nuño, and J. Martinez, IEEE Trans. Power Electron. 23, 1608 (2008). 